\title{
Self-assembled morphologies of monotethered polyhedral oligomeric silsesquioxane nanocubes from computer simulation
}

\author{
Xi Zhang \\ Department of Materials Science and Engineering, University of Michigan, Ann Arbor, \\ Michigan 48109-2136 \\ Elaine R. Chan \\ Department of Chemical Engineering, University of Michigan, Ann Arbor, Michigan 48109-2136 \\ Sharon C. Glotzer ${ }^{\mathrm{a})}$ \\ Department of Materials Science and Engineering and Department of Chemical Engineering, \\ University of Michigan, Ann Arbor, Michigan 48109-2136
}

(Received 22 June 2005; accepted 16 August 2005; published online 10 November 2005)

\begin{abstract}
Self-assembly of functionalized nanoscale building blocks is a promising strategy for "bottom-up" materials design. Recent experiments have demonstrated that the self-assembly of polyhedral oligomeric silsesquioxane (POSS) "nanocubes" functionalized with organic tethers can be utilized to synthesize novel materials with highly ordered, complex nanostructures. We have performed molecular simulations for a simplified model of monotethered POSS nanocubes to investigate systematically how the parameters that control the assembly process and the resulting equilibrium structures, including concentration, temperature, tether lengths, and solvent conditions, can be manipulated to achieve useful structures via self-assembly. We report conventional lamellar and cylindrical structures that are typically found in block copolymer and surfactant systems, including a thermotropic order-order transition, but with interesting stabilization of the lamellar phase caused by the bulkiness and cubic geometry of the POSS nanocubes. (C) 2005 American Institute of Physics.
\end{abstract} [DOI: $10.1063 / 1.2060664]$

\section{INTRODUCTION}

Self-assembly of nanoscale molecules and particles into nanostructures is a promising strategy for "bottom-up" materials design. ${ }^{1,2}$ A remarkable variety of nanoscale building blocks (NBBs), including rods, ${ }^{3}$ cubes, ${ }^{4}$ tetrapods, ${ }^{5}$ centipedes, ${ }^{6}$ triangular frames, ${ }^{7}$ and hexagons ${ }^{8}$ are available today. These NBBs can be functionalized with synthetic and biological molecules to enable the control of assembled nanostructures and ultimately the design of nanomaterials and devices with novel or enhanced electrical, optical, rheological, thermal, and mechanical properties. Polyhedral oligomeric silsesquioxane (POSS) molecules are a class of cubic-shaped NBBs that can self-assemble to construct hybrid organic/inorganic nanomaterials with superior physical properties. ${ }^{9}$ POSS nanocubes consist of silicon and oxygen atoms linked into a well-defined, cubic inorganic framework with silicon atoms at the corners and oxygen atoms interspersed along the edges [Fig. 1(a)]. Each of the silicon corners can be functionalized with a variety of organic substituents ${ }^{10}$ to confer precise control over the topology and architecture of the NBB and the resulting self-assembled nanostructures. In particular, one of the cube corners can be functionalized with organic polymer chains or tethers to form monotethered POSS nanocubes, and some interesting morphologies have been reported from their self-assemblies. For

\footnotetext{
${ }^{a)}$ Author to whom correspondence should be addressed. Electronic mail: sglotzer@umich.edu
}

example, Knischka et al. ${ }^{11}$ attached poly(ethylene oxide) tethers to $\mathrm{H}$ - and $\mathrm{CH}_{3} \mathrm{CH}_{2}$-functionalized POSS nanocubes and observed micelles and vesicles in aqueous solutions. Kim et al. ${ }^{12}$ synthesized POSS molecules with a polyoxazoline tether and also reported micellar aggregates formed by the NBBs in aqueous solution. Cardoen and Coughlin ${ }^{13}$ prepared POSS molecules with a polystyrene tether and observed alternating layers of POSS and polystyrene in organic solvent, which suggests a lamellar or cylindrical morphology. Leu et al. ${ }^{14}$ investigated the self-assembly of polyimidetethered POSS molecules and observed zigzag-shaped cylinders or lamellae in transmission electron micrographs.

Although these experiments have demonstrated the feasibility of material design through self-assembly from functionalized POSS NBBs, much of the vast parameter space of such complex assemblies remains unexplored. Moreover, a comprehensive and predictive design scheme has yet to be formulated, and this motivates the present work. We can loosely consider functionalized NBBs as block copolymer analogs with the NBBs as one block and tethers as the other block, or as surfactant molecules with the NBBs as head groups and tethers as tail groups. ${ }^{15}$ Hence we expect them to share some common features with these other amphiphilic systems. Generally, noncovalent, weak intermolecular interactions are responsible for the immiscibility-induced ordering in soft materials, and the interfacial curvature induced by intermolecular packing is responsible for the geometry of their microphase-separated domains. ${ }^{2}$ Specifically, intermolecular interactions between block copolymers are character- 


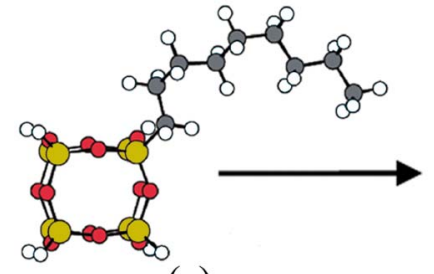

(a)

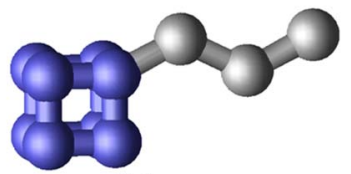

(b)
FIG. 1. Atomistic representation (a) and simplified model (b) of a monotethered POSS molecule $\mathrm{CH}_{3}\left(\mathrm{CH}_{2}\right)_{8} \mathrm{H}_{7} \mathrm{Si}_{8} \mathrm{O}_{12}$. In the atomistic representation, the silicon atoms are shown in maize, carbon atoms in gray, oxygen atoms in red, and hydrogen atoms in white. In the simplified model, the POSS nanocube (blue) is represented by eight beads connected by perfectly rigid bonds, and the polymer tether (gray) is represented by a bead-spring model with beads connected by FENE springs.

ized by the Flory-Huggins parameter $(\chi)$ and segment length $(N)$. Their product $(\chi N)$ is used to predict the order-disorder transition. The interfacial curvature resulting from the different stretching penalties of the blocks can be written as a function of block fraction $(f)$. Therefore $f$ can be used to predict the microstructural geometries and domain sizes in ordered phases for block copolymers in the strong segregation limit. ${ }^{16}$ For surfactant systems, the Israelachvili packing factor characterizes the average shape of aggregated amphiphilic molecules and is used to geometrically rationalize the microphase-separated structures. ${ }^{17}$

Computational studies can help predict structures and efficiently map the parameter space and provide fundamental insight into the various assembly processes. Computer simulations have been successfully employed to study the selfassembled structures and phase diagrams of block copolymer ${ }^{18}$ and surfactant systems. ${ }^{19}$ Recently, Zhang et $a l .{ }^{15}$ developed a general coarse-grained model of tethered NBBs and performed molecular simulations to study assemblies of tethered NBBs with a variety of topologies and geometries. Similarly, Chan et al. ${ }^{20}$ developed a simple minimal model of tethered POSS cubes to study the selfassembly of tetratethered POSS via computer simulation and mapped the morphological phase diagrams. Here we present a computational study of monotethered nanocube assemblies using similar methodologies. We investigate the effects of volume fraction, solvent quality, temperature, concentration, and tether lengths on the final assembled structures. Comparing our results with the self-assembled microphases of block copolymer and surfactant systems, we demonstrate that the above-mentioned general principles of soft matter assemblies can be extended to the monotethered POSS system. However, we find that the bulkiness and cubic geometry of our NBBs dominate the intermolecular packing for certain solvent conditions or cube-cube interactions, and hence alter the assembled equilibrium mesoscale structures from those exhibited by surfactants and diblock copolymers.

\section{METHODOLOGY}

\section{A. Simulation model}

In this work, we adopt a coarse-grained model developed for mesoscale simulation of tethered NBB self-assembly. ${ }^{15,20}$ We model the cubic inorganic cage of the
NBB as a rigid cube comprised of eight beads, where each bead represents one corner group of the POSS nanocube. With the rigid cube approximation we can treat one $\mathrm{Si}_{8} \mathrm{O}_{12}$ cage as a single entity with eight interaction sites instead of 20 individual atoms, resulting in fewer force calculations. By ignoring the high-frequency intracage vibrations, larger time steps and thus longer time and length scales may be realized. The rigid cube model is supported by experimental studies that demonstrate less than $5 \%$ deformation in the $\mathrm{Si}_{8} \mathrm{O}_{12}$ cages in alkyl-functionalized POSS crystals. ${ }^{21}$ Moreover, $a b$ initio density-functional theoretical calculations have revealed very little deformation in POSS NBBs with polymer tethers attached. ${ }^{20}$ The rigid cube approximation has been employed in simulations of octafunctionalized POSSpolymer networks which successfully reproduced physical properties observed in experiments. ${ }^{22}$ A similar model was used to study tetratethered POSS molecules and map the phase diagrams of POSS NBBs with various tether lengths. ${ }^{20}$

As in Ref. 20, we model the tether attached to each POSS molecule as a bead-spring chain [Fig. 1(b)] with neighboring beads connected by finitely extensible nonlinear elastic $^{23}$ (FENE) potential

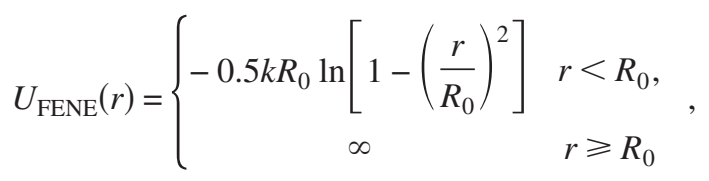

where $r$ is the separation between two bonded beads, $k$ is the spring constant, and $R_{0}$ is the maximum bond length. We choose $k=30$ and $R_{0}=1.5 \sigma$ to ensure relatively stiff bonds while avoiding very high-frequency modes and chain crossing.

We model selectivity of the implicit solvent for a specific bead type (i.e., POSS nanocube or tether) by specifying different nonbonded interactions between those bead types. In a poor solvent, the beads are effectively attracted to each other and this behavior is modeled by the truncated and shifted Lennard-Jones (LJ) potential ${ }^{24}$

$$
\begin{aligned}
U_{\mathrm{LJ}}(r)= & 4 \varepsilon\left[\left(\frac{\sigma}{r}\right)^{12}-\left(\frac{\sigma}{r}\right)^{6}-\left(\frac{\sigma}{r_{c}}\right)^{12}+\left(\frac{\sigma}{r_{c}}\right)^{6}\right] \\
& -\left(r-r_{c}\right)\left(\frac{d U_{\mathrm{LJ}}(r)}{d r}\right)_{r=r_{c}} r \leqslant r_{c},
\end{aligned}
$$

where $r$ is the distance between two beads and $r_{c}=2.5 \sigma$ is the cutoff radius. In good solvent, the beads are effectively repelled by each other and these short-range interactions are modeled by the Weeks-Chandler-Andersen (WCA) softsphere potential ${ }^{25}$

$$
U_{\mathrm{WCA}}(r)=4 \varepsilon\left[\left(\frac{\sigma}{r}\right)^{12}-\left(\frac{\sigma}{r}\right)^{6}\right]+\varepsilon \quad r \leqslant r_{c},
$$

where $r_{c}=2^{1 / 6} \sigma$ is the cutoff radius. The immiscibility between different bead types is also treated via this purely repulsive interaction. 
TABLE I. Summary of simulation runs performed.

\begin{tabular}{|c|c|c|c|c|c|}
\hline Solvent condition & Tether length & \#NBBs & $\phi$ & $T^{*}$ & Morphology \\
\hline Neutrally poor & $4,6,8,10,12,14$ & 3000 & 0.4 & 1.5 & lamellae \\
\hline Neutrally poor & 4 & 3000 & 0.3 & 1.5 & disordered \\
\hline Neutrally poor & $6,8,10,12,14$ & 3000 & 0.3 & 1.5 & lamellae \\
\hline Neutrally poor & $16,18,20,24$ & 1000 & 0.3 & 1.5 & lamellae \\
\hline Neutrally poor & $16,18,20,24$ & 2000 & 0.3 & 1.5 & lamellae \\
\hline Poor for cube & $4,6,8,10,12$ & 3000 & 0.3 & 1.3 & lamellae \\
\hline Poor for tether & 6 & 3000 & 0.3 & 1 & cylinder \\
\hline Poor for tether & $8,10,12$ & 1000 & 0.3 & 1 & cylinder \\
\hline Poor for tether & 6 & 3000 & 0.3 & 1.5 & disordered \\
\hline Poor for tether & 8,10 & 1000 & 0.3 & 1.5 & disordered \\
\hline Poor for tether & $12,14,20,24$ & 1000 & 0.3 & 1.5 & lamellae \\
\hline Poor for tether & $12,14,16,18,20,24$ & 2000 & 0.3 & 1.5 & lamellae \\
\hline
\end{tabular}

\section{B. Simulation details}

We employ Brownian dynamics, a stochastic molecular dynamics simulation method ${ }^{26}$ in which the equation of motion for each bead $i$ is

$$
m_{i} \dot{\mathbf{v}}_{i}(t)=-m_{i} \xi_{i} \mathbf{v}_{i}(t)+\mathbf{F}_{i}\left(\mathbf{x}_{i}(t)\right)+\mathbf{R}_{i}(t)
$$

where $m_{i}, \mathbf{x}_{i}, \mathbf{v}_{i}, \mathbf{F}_{i}$, and $\xi_{i}$ are the mass, position, velocity, force, and friction coefficient of bead $i$, respectively. The friction term acts as a heat sink while the random term $\mathbf{R}_{i}$ acts as a heat source and is calculated from the fluctuationdissipation theorem ${ }^{27}$

$$
\left\langle\mathbf{R}_{i}(t) \mathbf{R}_{j}\left(t^{\prime}\right)\right\rangle=6 m \xi k_{B} T \delta_{i j} \delta\left(t-t^{\prime}\right) .
$$

The friction and random forces effectively couple the system to a heat bath, and hence these simulations sample a canonical (NVT) ensemble, but without momentum conservation.

We use LJ reduced units in which the basic units for length and energy are $\sigma$ and $\varepsilon$, respectively. Each bead has the same size $\sigma=1$ and mass $m=1$. The reduced temperature is defined as $T^{*}=k_{B} T / \varepsilon$, the reduced time step as $\Delta t$ $=t / \sigma \sqrt{m / \varepsilon}$, and the reduced friction coefficient as $\xi^{*}$ $=\xi \sigma \sqrt{m / \varepsilon}$. We use the leap-frog algorithm ${ }^{24}$ with a time step $\Delta t=0.01$ to integrate the equations of motion. We choose $\xi^{*}=1$ to simulate systems in the range between overdamped and purely deterministic regimes. The overall volume fraction of the system is given by $\phi=\pi \sigma^{3} N / 6 V$, where $N$ denotes the total number of beads and $V$ is the volume of the simulation box. The rotational degrees of freedom of the rigid cubes are treated using the method of quaternions. ${ }^{24}$ Each system in our simulations is initially relaxed athermally (i.e., with only excluded volume interactions) into a disordered configuration and subsequently cooled to a chosen target temperature. Both cooling and heating processes are carried out to study the order-order transition (OOT). We first cool the system from high temperature $\left(T^{*}=1.5\right)$ to low temperature $\left(T^{*}=1.0\right)$ in small increments $\left(\Delta T^{*} \leqslant 0.125\right)$, equilibrating at each intermediate state point, and then reverse the path by heating the systems again in small increments $\left(\Delta T^{*} \leqslant 0.125\right)$. The OOT is located by examining nonbonded potential energies as a function of $T^{*},{ }^{28}$ and by inspecting the equilibrium structure at each $T^{*}$.
In a typical simulation, 3000 POSS molecules with tethers of a certain length are placed into a cubic simulation box with periodic boundary conditions. Based on previous work, ${ }^{29}$ we do not expect finite-size effects to be an important factor for our simulation sizes since lamellar and cylindrical structures are the only ordered phases studied here. These two ordered phases possess infinite wave vectors in fewer than three dimensions, and can therefore orient themselves within the simulation box so that the finite wave vectors of the pattern nearly match those of infinite bulk samples. ${ }^{30}$ To verify this, we carry out simulations at the same state point with 1000, 2000, and 3000 molecules, and monitor the potential energy and the stress tensor. We find that each of these quantities is identical in systems of different sizes, implying that the finite box sizes do not affect the assembled equilibrium phases reported here. A summary of the simulation runs performed is presented in Table I.

\section{RESULTS AND DISCUSSION}

We investigate the structures resulting from selfassembly of monotethered POSS nanocubes under various concentrations, temperatures, tether lengths, and solvent conditions, and compare the phase behaviors of these systems with block copolymers and surfactants.

\section{A. Order-disorder transitions (ODTs)}

Figures 2(a) and 2(b) show snapshots of equilibrium configurations of POSS nanocubes with tether lengths of four and six beads at overall volume fractions $\phi=0.3$ and 0.4 in neutrally poor solvent at $T^{*}=1.5$. At this temperature, ordered lamellar structures are found at overall volume fraction $\phi=0.3$ with tethers of six beads and longer [Fig. 2(a)], or at overall volume fraction $\phi=0.4$ for tethers of length four beads or longer [Fig. 2(b)]. Disordered configurations are found as the tether length decreases from six to four beads or as the volume fraction decreases from 0.4 to 0.3 (Table I). This order-disorder transition (ODT) can be understood via analogy with block copolymers. A shorter tether length implies shorter block length and fewer segments $(N)$, while lower volume fraction reduces the effective bead-bead interactions, thereby decreasing the Flory-Huggins parameter $(\chi)$. 


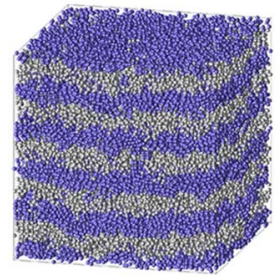

(a)

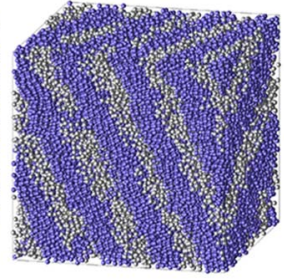

(b)

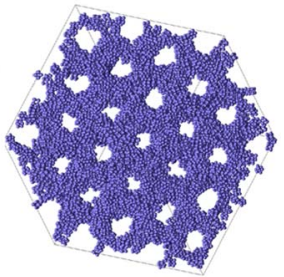

(c)

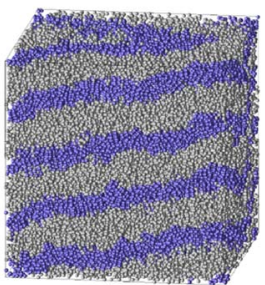

(a)

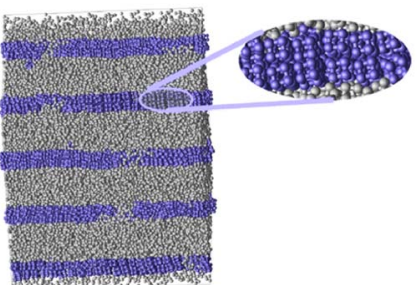

(b)

FIG. 2. Equilibrium configurations of (a) six-bead tethered POSS nanocubes at overall volume fraction $\phi=0.3$ and (b) four-bead tethered POSS nanocubes at overall volume fraction $\phi=0.4$, both in neutrally poor solvent at $T^{*}=1.5$. (c) Six-bead tethered POSS nanocubes at overall volume fraction $\phi=0.3$ in selective solvent that is good for POSS nanocubes and poor for tethers at $T^{*}=1.0$ (for clarity, only the POSS nanocubes are shown in the snapshot)

This trend in the ODT with decreasing $\chi N$ is consistent with the ODT behavior observed in block copolymers. ${ }^{16}$

Decreasing the temperature also increases $\chi$ and induces an ODT. Figure 2(c) shows a hexagonal cylinder structure of POSS nanocubes with six-bead tethers at overall volume fraction $\phi=0.3$ in selective solvent that is good for POSS nanocubes and poor for tethers at $T^{*}=1.0$. Disordered configurations are found at higher temperatures (Table I). This temperature-driven ODT is also consistent with the ODT behavior of block copolymers. ${ }^{16}$

\section{B. Stabilized lamellar structures in solvents poor for POSS nanocubes}

We simulate the self-assembly of POSS nanocubes with tether lengths ranging from 6 to 24 beads at fixed overall volume fraction $\phi=0.3$ and $T^{*}=1.5$ in neutrally poor solvent. Lamellar structures are observed in all the equilibrium configurations [see, for example, the simulation snapshot of 12bead tethered POSS nanocubes in Fig. 3(a)]. In analogy with block copolymers, we can calculate the tether block fraction $(f)$ of the monotethered POSS molecules. When the size of the nanocube is fixed and the tether length increases to as long as 24 beads, $f$ approaches 0.75 , characterizing a highly asymmetric molecule. Block copolymers typically form lamellar structures when the sizes of the two blocks are comparable, and form gyroid phases, hexagonally packed cylinders, and micelles when one block is longer than the other. ${ }^{16}$ Here we find that the lamellar structures are stabilized and become the equilibrium phase even in the very asymmetric case $f=0.75$.

We also simulate the self-assembly of POSS nanocubes with tether lengths ranging from 4 to 12 beads at overall volume fraction $\phi=0.3$ and $T^{*}=1.3$ in selective solvent that is good for tethers and poor for POSS. Again, lamellar structures are observed in the equilibrium configurations [see, for example, the simulation snapshot of 12-bead tethered POSS nanocubes in Fig. 3(b)]. Since the solvent is selectively good for the tether beads, the tether block is swollen and its effective fraction $f$ is larger than 0.6 in the 12-bead system. This $f$ value is beyond those corresponding to the lamellar phase region of typical block copolymers, ${ }^{16}$ but we still observe a lamellar phase in our simulated equilibrium configuration.

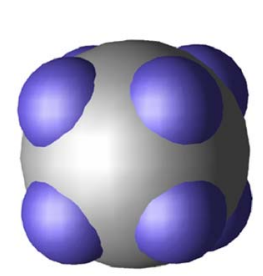

(c)

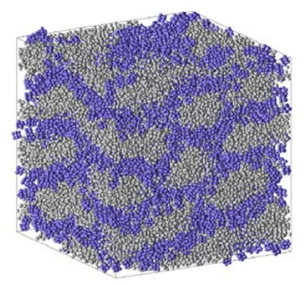

(d)

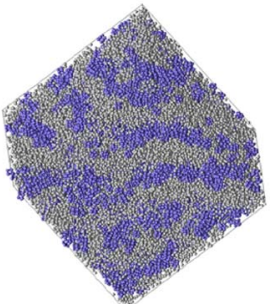

(e)
FIG. 3. Equilibrium configurations from 12-bead tethered POSS nanocubes at overall volume fraction $\phi=0.3$ (a) in neutrally poor solvent at $T^{*}=1.5$ and (b) in selective solvent that is poor for POSS nanocubes and good for tethers at $T^{*}=1.3$. (c) Cartoon model of a POSS nanocube superposed with a WCA repulsive site. Snapshots from 12-bead tethered POSS nanocubes superposed with WCA repulsive sites at overall volume fraction $\phi=0.3$ (d) in neutrally poor solvent at $T^{*}=1.5$ and (e) in selective solvent that is poor for POSS nanocubes and good for tethers at $T^{*}=1.3$.

The stabilization of lamellar phases can be explained by the face-to-face, intermolecular close packing induced by the cubic topology of POSS molecules, ${ }^{20}$ and the strong effective attractive interactions between POSS nanocubes in poor solvent. The simulation snapshots of the lamellar structures reveal that the POSS bilayers are composed of face-to-face closely packed nanocubes [inset in Fig. 3(b)]. Such face-toface close packing has been observed in octahydrido and octamethyl POSS crystals and monosubstituted $\mathrm{C}_{6} \mathrm{H}_{13}\left(\mathrm{H}_{7} \mathrm{Si}_{8} \mathrm{O}_{12}\right)$ crystals in experimental studies ${ }^{31}$ as well as atomistic simulations. ${ }^{32}$ We surmise that the strong faceto-face local packing favors flat interfaces between microphase-separated domains and appears to inhibit the formation of cylindrical morphologies with curved interfaces, thereby stabilizing the lamellar phase. To test this conjecture, we superpose a WCA interaction site over the mass center of each nanocube to inhibit their face-to-face local packing [Fig. 3(c)]. Figures 3(d) and 3(e) show the simulation snapshots of 12-bead tethered POSS nanocubes superposed with WCA repulsive sites simulated at the same state points as in Figs. 3(a) and 3(b), respectively. When the cubic face-to-face packing is sterically inhibited, some POSS nanocubes appear in the tether domain and the flat domain interfaces become frustrated, resulting in disruption of the lamellar structures. These results imply that the face-to-face local packing is essential for stabilizing the lamellar phases for highly asymmetric building blocks.

\section{Order-order transition (OOT) in selective solvent good for POSS nanocubes}

We simulate the self-assembly of POSS nanocubes with tether lengths ranging from 6 to 24 beads at $T^{*}=1.0$ and $T^{*}$ $=1.5$ at fixed overall volume fraction $\phi=0.3$ in selective sol- 


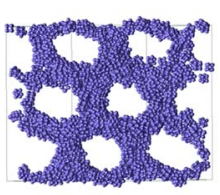

(a)

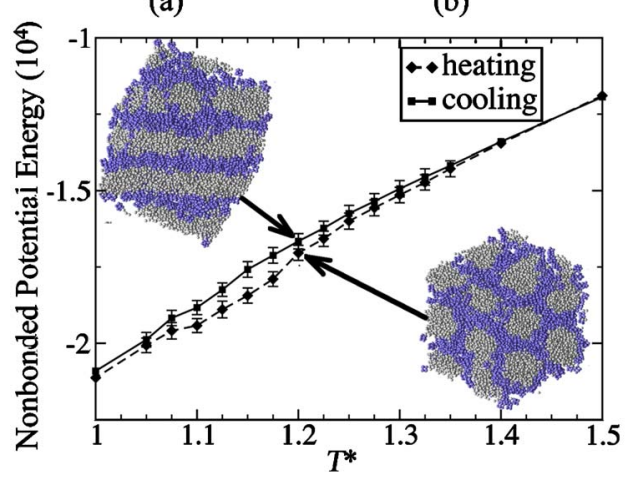

(c)

FIG. 4. Equilibrium configurations from 12-bead tethered POSS nanocubes at overall volume fraction $\phi=0.3$ in selective solvent that is good for POSS nanocubes and poor for tethers. (a) Cylinders at $T^{*}=1.0$ (for clarity, only POSS nanocubes are shown in the snapshot). (b) Lamellae at $T^{*}=1.5$. (c) Nonbonded interaction energy vs temperature during the cooling and heating processes. Insets: transition region snapshots at $T^{*}=1.2$ obtained upon heating the configuration in snapshot a (dashed line) and cooling the configuration in snapshot $b$ (solid line).

vent that is poor for tethers and good for POSS nanocubes. As discussed in Sec. III A, a temperature-driven ODT is observed as POSS nanocubes with six-bead tethers are heated from $T^{*}=1.0$ to $T^{*}=1.5$. In the ordered phase region, we find tether-rich cylinders assembled in a hexagonal arrangement within a POSS-rich matrix at lower temperature [see, for example, the equilibrium configuration of 12-bead tethered POSS nanocubes at $T^{*}=1.0$ in Fig. 4(a)]. At higher temperature and at the same volume fraction, we find lamellar structures with the nanocubes randomly packed in POSS-rich domains [see, for example, the equilibrium configuration of 12-bead tethered POSS nanocubes at $T^{*}=1.5$ in Fig. 4(b)]. These ordered structures and the temperature-driven cylinder-lamellae OOT are consistent with self-consistent field theory calculations of diblock copolymer phase behavior in selective solvents. ${ }^{33}$ We further investigate the OOT of tethered POSS nanocubes by monitoring the interaction energies as a function of temperature. Figure 4(c) shows the nonbonded interaction energy versus temperature during the cooling and heating processes. We observe a weak hysteresis in the energy, which is characteristic of first-order transitions. In addition to the potential energy plot, inspection of the intermediate equilibrium snapshots during cooling and heating processes provides insight into the OOT pathway. Upon heating the cylindrical structures, the POSS domains between adjacent tether-rich cylinders dissolve and become progressively thinner as the temperature increases, eventually forming lamellae [see, for example, lower right inset in Fig. 4(c)]. Upon cooling these lamellar structures, an increasing number of POSS nanocubes appear in the tether domain and bridges form between layers until individual cylinders are eventually formed [see, for example, upper left inset in Fig. 4(c)].

\section{CONCLUSION}

We have employed a simplified molecular model to study the self-assembly of monotethered POSS molecules in neutral and selective solvents. We performed Brownian dynamics simulations with implicit solvent to study the phases formed by self-assembly. We have systematically explored the parameters that control the assembly process and the resulting equilibrium structures, including concentration, temperature, tether lengths, and solvent conditions. We observe the same ODT trends and similar lamellar and cylindrical structures in the POSS systems as are commonly found in surfactants and diblock copolymer systems. However, we find that strong face-to-face local packing, induced by the bulkiness and cubic geometry of POSS cages and their effective attractive interaction in poor solvent, stabilizes the lamellar structures and prohibits the formation of cylindrical structures with curved interfaces. We report a temperaturedriven OOT from lamellae at high temperature to ordered cylinders at low temperature in selective solvent that is good for POSS and poor for tethers. As found for block copolymers in selective solvents, the OOT may be a first-order transition.

\section{ACKNOWLEDGMENTS}

Financial support for this work has been provided by the National Science Foundation under Grant No. DMR0103399. E.R.C. acknowledges partial support from the Department of Education under the GAANN program. We are grateful for insightful conversations and collaboration with P.T. Cummings, J. Kieffer, C. McCabe, and M. Neurock as part of a larger effort to model POSS-polymer systems. ${ }^{34} \mathrm{We}$ thank E. B. Coughlin, R. M. Laine, and P. T. Mather for helpful discussion about their experiments. We thank the National Partnership for Advanced Computational Infrastructure (NPACI) and the University of Michigan Center for Advanced Computing for computer cluster support.

${ }^{1}$ G. M. Whitesides and M. Boncheva, Proc. Natl. Acad. Sci. U.S.A. 99, 4769 (2002).

${ }^{2}$ I. W. Hamley, Angew. Chem., Int. Ed. 42, 1692 (2003).

${ }^{3}$ N. R. Jana, L. A. Gearheart, S. O. Obare, C. J. Johnson, K. J. Edler, S. Mann, and C. J. Murphy, J. Mater. Chem. 12, 2909 (2002).

${ }^{4}$ Y. Sun and Y. Xia, Science 298, 2176 (2002).

${ }^{5}$ D. J. Milliron, S. M. Hughes, Y. Cui, L. Manna, J. Li, L.-W. Wang, and A. P. Alivisatos, Nature (London) 430, 190 (2004).

${ }^{6}$ Y. Wang, Z. Tang, X. Liang, L. M. Liz-Marzán, and N. A. Kotov, Nano Lett. 4, 225 (2004).

${ }^{7}$ G. S. Métraux, Y. C. Cao, R. Jin, and C. A. Mirkin, Nano Lett. 3, 519 (2003).

${ }^{8}$ T. D. Clark, R. Ferrigno, J. Tien, K. E. Paul, and G. M. Whitesides, J. Am. Chem. Soc. 124, 5419 (2002).

${ }^{9}$ J. D. Lichtenhan, Comments Inorg. Chem. 17, 115 (1995); J. J. Schwab and J. D. Lichtenhan, Appl. Organomet. Chem. 12, 707 (1998); R. M. Laine, C. Zhang, A. Sellinger, and L. Viculis, Appl. Organomet. Chem. 12, 715 (1998).

${ }^{10}$ F. J. Feher and T. A. Budzichowski, Polyhedron 14, 3239 (1995); C. Marcolli and G. Calzaferri, Appl. Organomet. Chem. 13, 213 (1999); G. Li, L. Wang, H. Ni, and C. U. Pittman, J. Inorg. Organomet. Polym. 11, 123 (2001).

${ }^{11}$ R. Knischka, F. Dietsche, R. Hanselmann, H. Frey, R. Mülhaupt, and P. J. Lutz, Langmuir 15, 4752 (1999).

${ }^{12}$ K.-M. Kim, D.-K. Keum, and Y. Chujo, Macromolecules 36, 867 (2003).

${ }^{13}$ G. Cardoen and E. B. Coughlin, Macromolecules 37, 5123 (2004).

${ }^{14}$ C.-M. Leu, G. M. Reddy, K.-H. Wei, and C.-F. Shu, Chem. Mater. 15, 
2261 (2003)

${ }^{15}$ Z. L. Zhang, M. A. Horsch, M. H. Lamm, and S. C. Glotzer, Nano Lett. 3, 1341 (2003).

${ }^{16}$ M. W. Matsen and F. S. Bates, Macromolecules 29, 1091 (1996).

${ }^{17}$ J. N. Israelachvili, Intermolecular and Surface Forces. (Academic, London, 1992).

${ }^{18}$ A. J. Schultz, C. K. Hall, and J. Genzer, J. Chem. Phys. 117, 10329 (2002).

${ }^{19}$ R. G. Larson, J. Phys. II 6, 1441 (1996).

${ }^{20}$ E. R. Chan, X. Zhang, C.-Y. Lee, M. Neurock, and S. C. Glotzer, Macromolecules 38, 6168 (2005).

${ }^{21}$ A. M. Bieniok and H. B. Bürgi, J. Phys. Chem. 98, 10735 (1994).

${ }^{22}$ M. H. Lamm, T. Chen, and S. C. Glotzer, Nano Lett. 3, 989 (2003).

${ }^{23}$ G. S. Grest and K. Kremer, Phys. Rev. A 33, 3628 (1986).

${ }^{24}$ M. P. Allen and D. J. Tildesley, Computer Simulation of Liquids. (Clarendon, Oxford, 1987).

${ }^{25}$ J. D. Weeks, D. Chandler, and H. C. Andersen, J. Chem. Phys. 54, 5237 (1971).
${ }^{26}$ W. F. van Gunsteren, H. J. C. Berendsen, and J. A. C. Rullmann, Mol. Phys. 44, 69 (1981); G. S. Grest, M. D. Lacasse, K. Kremer, and A. M. Gupta, J. Chem. Phys. 105, 10583 (1996).

${ }^{27}$ R. Kubo, Rep. Prog. Phys. 29, 255 (1966).

${ }^{28}$ R. G. Larson, J. Chem. Phys. 96, 7904 (1992); H. Guo and K. Kremer, J. Chem. Phys. 119, 9308 (2003).

${ }^{29}$ M. A. Horsch, Z. L. Zhang, C. R. Iacovella, and S. C. Glotzer, J. Chem. Phys. 121, 11455 (2004).

${ }^{30}$ R. G. Larson, Chem. Eng. Sci. 49, 2833 (1994).

${ }^{31}$ T. P. E. Auf der Heyde, H.-B. Bürgi, H. Bürgy, and K. W. Törnroos, Chimia 45, 38 (1991); K. Larsson, Ark. Kemi 16, 203 (1960); G. Calzaferri, R. Imhof, and K. W. Törnroos, J. Chem. Soc. Dalton Trans. 1994, 3123.

${ }^{32}$ T. C. Ionescu, F. Qi, C. McCabe, A. Striolo, J. Kieffer, and P. T. Cummings, J. Phys. Chem. B (to be published).

${ }^{33}$ C. I. Huang and T. P. Lodge, Macromolecules 31, 3556 (1998).

${ }^{34}$ C. McCabe, S. C. Glotzer, J. Kieffer, M. Neurock, and P. T. Cummings, J. Comput. Theor. Nanosci. 1, 265 (2004). 